\section{Estructura y recursos humanos en un hospital público chileno: una experiencia de cambio}

\author{
Osvaldo Artaza Barrios, ${ }^{1}$ \\ Julio Montt Vidal ${ }^{2} y$ \\ Claudia Vásquez Leichtle ${ }^{3}$
}

\section{RESUMEN}

En este artículo se narra la reforma llevada a cabo desde 1994 en adelante en la estructura y el funcionamiento del hospital Luis Calvo Mackenna, centro asistencial pediátrico de nivel terciario perteneciente al Servicio de Salud Metropolitano Oriente, de Santiago, Chile. Se refieren brevemente los antecedentes de la formación del sistema de salud de Chile, los cambios traídos por el gobierno militar en 1979 y otras transformaciones debidas al primer gobierno democrático en 1990 y al segundo, establecido en 1994. A pesar de los buenos indicadores de salud de Chile y del deseo del gobierno actual de establecer equidad en los servicios y poner en marcha un sistema descentralizado, el sistema de salud modelado fundamentalmente en los decenios de 1950 y 1960 no ha podido hacer frente a los cambios causados por la transición demográfica y el nuevo perfil epidemiológico de la población. Uno de los grandes problemas es que los niveles secundario y terciario de atención no tienen la capacidad necesaria para responder a la demanda. Bajo una nueva dirección interesada en métodos contemporáneos de gestión, en el hospital Luis Calvo Mackenna se reestructuraron los servicios clínicos con nuevos departamentos y unidades, se llevó a cabo un estudio del clima organizacional, se capacitó intensivamente a todo el personal para inculcarle el espíritu de participación y una nueva visión de la misión del hospital y del trabajo en equipo, y se formuló un plan estratégico que todavía se está cumpliendo.

\footnotetext{
1 Hospital Luis Calvo Mackenna. Dirección postal: Ministerio de Salud, Servicios de Salud Metropolitano Oriente, Antonio Varas 360, Providencia, Casilla 16260, Correo 9, Santiago, Chile.

2 Servicio de Salud Metropolitano Oriente, Santiago, Chile.

3 Hospital Luis Calvo Mackenna, Unidad de Desarrollo y Estudios, Santiago, Chile.
}

Chile tiene una vasta tradición de experiencia en salud pública y el Estado siempre ha tenido un papel destacado en las políticas y ejecución de programas de salud de amplia cobertura. En los años cincuenta, la creación del Servicio Nacional de Salud (SNS) a partir de la fusión de diversas entidades de beneficencia permitió el desarrollo de un sistema público de salud con una infraestructura hospitalaria importante e hizo posibles proyectos y programas de impacto considerable. Entre las actividades emprendidas entonces sobresalen las de vacunación, control de la salud del niño y de la embarazada, y atención del parto. Durante los 40 años siguientes, las condiciones generales de vida en el país mejoraron y las prestaciones del sistema público se extendieron a una población cada vez más numerosa. Para 1996 la mortalidad infantil había disminuido de más de 120 a menos de 11 por 1000 nacidos vivos, mientras que la atención profesional del parto cubría a más de $97 \%$ de la población objetivo.

\section{La reforma de 1979}

En 1979, el gobierno militar llevó a cabo una profunda reforma del sector de la salud. Se suspendió el financiamiento estatal directo de los servicios de salud y se creó el sistema de seguros privados conocido como Instituciones de Salud Previsional (ISAPRES), que potenció el surgimiento vigoroso de una infraestructura de carácter privado. Actualmente ese subsector privado atiende a $25 \%$ de la población, por concepto de unos US\$ 300 per cápita al año. Se han generado así algunas inequidades evidentes, ya que el sector público gasta solo $\$ 200$ per cápita. También ha habido un aumento preocupante del costo de la atención médica a la vez que muchos usuarios se quejan de la insuficiente cobertura de los programas, de la selección negativa de los afiliados de mayor riesgo y de problemas aún no resueltos en relación con el cuidado de pacientes de la tercera edad y las enfermedades de costo extremado o catastróficas que debe ser asumido por el sector público, el cual se comporta como un seguro.

En segundo lugar, con la reforma se procuró descentralizar y regionalizar los servicios eliminando el SNS y creando en su lugar el Sistema Nacional de Servicios de Salud. La antigua Dirección General del SNS dio origen a 27 servicios con 
recursos financieros y humanos propios. Toda la atención primaria pasó a estar bajo la jurisdicción de los más de 300 municipios del país (1981-1989), lo que produjo en la red asistencial una desintegración que todavía no se ha llegado a evaluar muy bien.

La introducción de un estricto esquema económico neoliberal impuso una fuerte restricción del gasto público. En consecuencia, cesaron las inversiones en el sector de la salud y perdieron sus trabajos y poder adquisitivo numerosos empleados públicos. Sin embargo, la reforma del gobierno militar no alcanzó el marco jurídico y reglamentario de gestión de los recursos humanos. Se mantuvo vigente la ley 15.076 que rige la contratación de médicos, bioquímicos, quimicofarmacéuticos y odontólogos y se dictó un nuevo estatuto administrativo que conservaba el sistema clásico de contratación centralista y rígido sin incentivos, que fomenta la inamovilidad de los funcionarios.

\section{Los gobiernos democráticos}

En 1990, a la llegada del primer gobierno democrático, el sector público de la salud se encontraba seriamente deteriorado. El equipamiento médico estaba obsolescente; la infraestructura física (calderas, cañerías para el agua, alambrado eléctrico y otros sistemas) había llegado a un punto crítico de deficiencia, y el escaso personal estaba desmoralizado. El sector privado había atraído al personal más capacitado ofreciendo mejores salarios y perspectivas de trabajo, y la situación se hacía especialmente difícil por la pérdida de muchas enfermeras universitarias, que en Chile asumen diversas funciones médicas en los hospitales públicos.

Ese primer gobierno democrático centró su quehacer en revertir la situación crítica del sector público de la salud aumentando la inversión de fondos de 3 a 5\% del producto interno bruto, implantando un programa de equipamiento médico, normalizando los sistemas hospitalarios y creando nuevos establecimientos, de atención secundaria en particular. La decisión de invertir en la infraestructura en vez de mejorar los salarios generó gran descontento en el colegio médico, luego una huelga de los servicios de urgencia del país y, por último, la caída del ministro de salud. Mientras que el personal médico atribuyó la crisis a que el sistema público no era capaz de brindarles los recursos necesarios para atender apropiadamente a la población, la gente vio en ella los resultados de una gestión inepta y asignación de recursos inadecuada para atender a sus demandas.

El actual gobierno democrático establecido en 1994 busca modernizar profundamente el sector de la salud en el sentido de establecer equidad en los servicios; reformar las prácticas de financiamiento, gestión y manejo del recurso humano; conseguir mayor participación de los actores, y poner en marcha un sistema descentralizado. Los indicadores de salud en Chile son excelentes, probablemente gracias a los progresos en el nivel de vida. No obstante, en los años noventa se está enfrentando una grave crisis de oferta y demanda en el área de la atención de salud. El modelo de sistema público de salud desarrollado en los decenios de 1950 y 1960 ha tenido que hacer frente a cambios causados por la transición demográfica en la estructura de la población y en su perfil epidemiológico. Este perfil, con causas principales de muerte que incluyen las enfermedades cardiovasculares, cáncer y traumatismos, se asemeja al de los países desarrollados. Además, han aumentado las expectativas y exigencias de los usuarios con respecto a la calidad de la atención. La atención primaria está mal articulada con los servicios, no cuenta con suficientes recursos y no se ha reorientado debidamente a los nuevos retos epidemiológicos. En cuanto a los niveles secundario y terciario, no tienen la capacidad para responder adecuadamente a la demanda. La resolución de estos problemas en el ámbito nacional exigirá un gran esfuerzo continuo de gestión, dotación de recursos humanos y cambios en la cultura y estructura organizacionales.

Dados los problemas descritos, en 1994 la dirección del hospital Luis Calvo Mackenna decidió iniciar profundas reformas que se llevarían a cabo en cuatro fases: 1) reestructuración de los servicios clínicos; 2) estudio del clima organizacional; 3) cambio hacia una visión, misión y gestión compartidas por todo el personal, y 4) ejecución de un plan estratégico institucional, que todavía se está llevando a cabo.

\section{El hospital Luis Calvo Mackenna}

Fundado en 1942, el hospital estatal Luis Calvo Mackenna es un centro asistencial pediátrico de nivel terciario, que brinda atención especializada, de urgencia y de hospitalización quirúrgica y médica general. Es un centro de referencia nacional para cirugía cardiovascular, oncología, cirugía neonatal, escoliosis, nefrourología y trasplantes. Pertenece al Servicio de Salud Metropolitano Oriente, uno de los 27 servicios descentralizados que integran el sistema público de salud de Chile. El hospital tiene 240 camas, 95 de ellas para pacientes con trastornos médicos no quirúrgicos, 90 para pacientes de cirugía y 55 para los que necesitan cuidados intensivos e intermedios pediátricos, neonatales y posquirúrgicos. 
Las estadísticas de 1996 indican que durante ese año en el hospital hubo 20000 egresos (60\% por cirugía) y se realizaron 10000 procedimientos quirúrgicos mayores, 150000 consultas especializadas y 100000 consultas de urgencia. En promedio, la estancia fue de 4 días y el índice de ocupación, de 86\%. El hospital cuenta con 1015 funcionarios: 34 directivos; 120 médicos y 172 profesionales de colaboración (enfermeras universitarias, tecnólogos médicos, quinesiólogos), 102 administrativos, 162 auxiliares de servicio y 425 técnicos paramédicos (auxiliares de enfermería).

En 1996, el presupuesto mensual de operación del hospital alcanzó aproximadamente US\$ 1,5 millones, de cuya cantidad cerca de un millón correspondió a remuneraciones y $\$ 500000$ a bienes y servicios de consumo (\$300 000 en farmacia). Del total del presupuesto, las transferencias estatales financiaron $\$ 1150000$ y los ingresos propios, por venta de servicios a clientes privados, $\$ 350000$. El aporte de estos últimos ingresos ha aumentado en los últimos 4 años de 10 a 30\% del presupuesto total de operación como resultado del empeño de la actual dirección por fortalecer la imagen corporativa del hospital y su posición en el mercado; competir, hacer convenios e integrarse con el sector privado; implantar mejores sistemas de registro y cobranza, y aprovechar la infraestructura ociosa. Al mismo tiempo, el Estado ha aumentado su aporte en $80 \%$, mientras que los ingresos propios aumentaron en $180 \%$, sin incrementar grandemente el número de pacientes privados, sino fundamentalmente por el mejor sistema de cobro y precios adecuados. El hospital tiene una gestión más profesional y hábil y ha adquirido estabilidad financiera. Su buena capacidad de pago facilita la negociación con proveedores. Su producción es el doble de lo que era en 1993 y, además, se han iniciado numerosos programas y proyectos en los que participa la comunidad. ${ }^{4}$

Cabe observar que en los últimos 4 años cerca de $40 \%$ de la inversión en nuevas estructuras físicas y equipo ha provenido de aportes de la comunidad por conducto de corporaciones de derecho privado sin fines de lucro equivalentes a las organizaciones para recaudación de fondos que existen en los Estados Unidos de América. La más importante de

\footnotetext{
Ejemplos de programas: manejo del paciente crónico en la comunidad y plan de oxígeno en el domicilio; agrupaciones de padres de pacientes con enfermedades crónicas; programas comunitarios de apoyo escolar, psicológico y recreativo para el niño hospitalizado; casa de huéspedes para recibir a niños y sus padres que vienen de regiones apartadas; sensibilización de jóvenes escolares mediante su colaboración con el hospital (visitas al hospital, recaudación de fondos en escuelas y tareas de apoyo a los niños hospitalizados); y campañas públicas de recaudación de fondos con participación de la comunidad y de los medios de comunicación (en 4 años, los grupos Amigos del Calvo Mackenna y Salvémos el Corazón al Niño recaudaron US $\$ 5$ millones y $\$ 3$ millones, respectivamente).
}

estas, Amigos del Calvo Mackenna (AMICAM), acaba de aportar $\$ 1,5$ millones para la construcción de ocho nuevos pabellones quirúrgicos. Otra de ellas, Salvemos el Corazón al Niño (SALVECOR), construyó con donaciones de empresas privadas un edificio anexo de dos pisos para cirugía cardíaca. En estos casos, la organización donante administra los nuevos servicios, pero el financiamiento y las aplicaciones técnicas son responsabilidad del hospital. Esta es una de las nuevas formas de gestión que se han explorado, en las que funcionan juntos exitosamente los sectores público y privado. En gran parte, la buena situación actual del hospital se debe también a que se han podido efectuar cambios y a la vez aprovechar las fortalezas conocidas. Se aprovecharon, por ejemplo, la imagen favorable de la institución en sentido técnico y académico, el personal calificado y la política de comunicación con la comunidad.

\section{Problemas principales de gestión en recursos humanos}

En los hospitales públicos de Chile, el problema central en recursos humanos puede resumirse brevemente: incapacidad para generar políticas efectivas de selección, capacitación, evaluación, reconocimiento y remuneración. Ese problema tiene sus antecedentes en un sistema legalizado que hace imposible ofrecer estímulo económico al personal, sea por buen desempeño, asistencia, sentido de responsabilidad $\mathrm{u}$ otras razones, pues las remuneraciones están fijadas por ley y son iguales para todos los funcionarios según criterios de grado y antigüedad solamente. Esto crea un ambiente que se expresa en la frase "el personal hace como que trabaja, ya que el Ministerio hace como que les paga". En los hospitales públicos de Chile el absentismo suele alcanzar $30 \%$ y la actitud general es de no esforzarse mucho para que los jefes no insistan en más trabajo aún. El personal no se siente motivado, ya que sus esfuerzos no se recompensan, y muchos pacientes se quejan de su trato. Además, existe un sistema de evaluación tan desvirtuado y obsoleto que cerca de $90 \%$ de los funcionarios están en la lista de méritos. Es prácticamente imposible despedir a un mal empleado, pues los funcionarios titulares $-80 \%$ de la planta- son casi dueños de sus puestos y se requiere comprobar, mediante 2 o 3 años de trámites, que alguien ha cometido delitos muy graves antes de poder despedirlo. Mientras tanto, le quedan disponibles numerosas formas de apelación.

Básicamente, el problema descrito se fundamenta en que la planta de cargos es fijada por ley. Las dotaciones de puestos y la carrera de grados y ascensos de cada funcionario son centralizadas e 
inflexibles. No dependen de una institución individual. Con un sistema tan rígido es imposible ofrecer contratos provisionales, de horario flexible o variaciones de salario según especialización, responsabilidad, realidad del mercado y otros factores; reforzar las unidades más críticas; o usar intensivamente la infraestructura las 24 horas del día.

Cualquier posible desarrollo de políticas efectivas de bienestar interno se ve coartado por las limitaciones impuestas sobre el manejo de los fondos de la institución. Todo lo anterior obstaculiza la posibilidad de reestructurar flexiblemente la organización de un hospital según las necesidades de los usuarios. Los servicios clínicos y administrativos, las jefaturas, y las dependencias jerárquicas y funcionales están determinados por numerosos reglamentos y ordenanzas gubernamentales.

Los salarios son inferiores a los que ofrece el subsector privado $\mathrm{y}$, en consecuencia, el personal mejor calificado migra constantemente en esa dirección. En la práctica, los hospitales públicos tienen que contentarse con lo que tienen, pues sin remuneraciones competitivas es imposible justificar una política de selección real del recurso humano.

\section{Cambio institucional por gestión innovadora}

Frente a los graves problemas de gestión en recursos humanos y otras áreas, la dirección del hospital decidió iniciar un proceso de cambio esencialmente cultural, cuyas directrices fundamentales serían: lograr una organización formal e informalmente centrada en el cliente; orientar a toda la organización hacia el cambio y realizarlo en forma participativa. Una vez lograda la estabilidad financiera del establecimiento, la ejecución del proceso de cambio empezó con la formulación de un plan estratégico institucional, al cual se sigue dedicando esfuerzo, especialmente en los aspectos fundamentales de capacitación y cambio gradual de estructura.

Uno de los principales problemas de estructura organizacional que presentaba el hospital era su configuración sumamente jerárquica, en la que el director - generalmente un médico con escasa formación gerencial - trabajaba aisladamente con la colaboración de un subdirector médico. De este subdirector dependían más de 20 jefes de servicios clínicos y unidades de apoyo, los cuales estaban más dedicados al desarrollo de sus especialidades que a la satisfacción de los usuarios. Este marco se prestaba habitualmente a fuertes mecanismos informales de comunicación, información y toma de decisiones.

El hospital contaba también con los servicios de un subdirector administrativo a cargo de los departamentos de finanzas, admisión, servicios generales, abastecimiento y farmacia. Este funcionario era, por lo general, una persona muy conservadora formada en las viejas tradiciones de la administración pública. Usualmente había grandes problemas de comunicación entre las áreas médica y administrativa del hospital.

La gestión del establecimiento se veía claramente entorpecida por esa estructura burocrática, poco estimada en el seno de la organización; la falta de definición de funciones; relaciones conflictivas que desanimaban el trabajo en equipo; y la opinión informal encubierta de que acaso ni era necesario tener un director. Por lo tanto, se apreció como prioridad revertir una situación tal que el hospital parecía desmembrado en partes poco coordinadas o solidarias; y el trabajo médico excesivamente enfocado en especialidades y aspectos académicos, con primacía de la introducción de tecnología y ultraespecialización. Hacía falta evaluar las actividades en función de los costos y evidentemente se desconocía la necesidad de determinar prioridades tanto sobre la base de pruebas científicas como sobre la de satisfacción del usuario. Todo ello, sumado al escaso interés en los problemas relacionados con la contención de gastos, mostraba la ausencia de una cultura de calidad.

Reestructuración de las unidades y servicios clínicos del hospital. Este proceso, que se inició a principios de 1994 antes de definir el plan estratégico general de la institución, cumplió a fines de 1996 tres años de realización progresiva. Constituyó un antecedente básico de la planificación y sirvió como plataforma en la definición de objetivos estratégicos dirigidos a mejorar la gestión hospitalaria. La reestructuración se planteó con el fin de reorganizar los servicios y las unidades clínicas en función de las necesidades y requerimientos de los usuarios o clientes. La filosofía básica era mejorar continuamente la calidad y dar capacidad, responsabilidad y poder a cada unidad para tomar decisiones sobre el uso de los recursos, es decir, procurar que se fueran convirtiendo poco a poco en unidades de autogestión clínica. En consecuencia, esas unidades pasaron a llamarse centros de responsabilidad.

Entrada esta primera fase, que implicó muchos conflictos, se estimó que era fundamental hacer un estudio del clima organizacional, como paso previo a la generación de un plan estratégico para la organización. En esta etapa se invirtió en capacitar a los miembros de la dirección de modo que se transformaran en un equipo de alto rendimiento capaz de definir e impulsar cambios profundos en el interior de la organización.

Para promover la descentralización, en la fase inicial de la reestructuración también se organizaron 
y activaron comités de apoyo a la gestión en farmacia, abastecimiento e infecciones nosocomiales. Se instauraron asimismo las bases de un sistema para la negociación de recursos sobre la base de resultados obtenidos. Además, se creó un departamento de enfermería encabezado por una enfermera coordinadora e integrado por las enfermeras jefas de capacitación y epidemiología hospitalaria, y enfermeras supervisoras en cada uno de los centros de responsabilidad. Las enfermeras pasaron así a desempeñar un papel importante en la gestión de los recursos, especialmente de los recursos humanos.

Estudio del clima organizacional. El estudio de clima permite objetivar algunos de los supuestos con que funciona el personal de una organización y cómo los clasifica en importancia en un momento dado. En el caso del hospital Luis Calvo Mackenna, se utilizó ese tipo de estudio para obtener información que era necesario considerar en la nueva orientación hacia la participación del personal en los cambios previstos, ya que permitiría visualizar la dinámica existente y dirigirse de forma más segura hacia el objetivo.

Para comprender los resultados del estudio, es necesario tener en cuenta la situación del hospital en ese momento. En primer lugar, el estudio se realizó en agosto de 1994, a los 6 meses de haberse establecido una nueva y joven dirección, en una organización no acostumbrada a ser liderada por el concepto contemporáneo de gestión. Como era de esperar, esta situación había generado partidarios y opositores, con comportamientos consecuentes que facilitaban o entorpecían los planes de la dirección. Por otra parte, debido a la prioridad asignada inicialmente a la estabilidad financiera y unicidad de criterios y objetivos, el estilo de gestión de la dirección estaba pasando por una fase de franco centralismo y escasa participación de los jefes de servicios y unidades en la toma de decisiones.

El estudio se realizó administrando a todo el personal del hospital un cuestionario anónimo cuyos resultados se dieron a conocer 4 meses después. Su divulgación constituyó un proceso de sensibilización para todos los empleados, en el sentido de que les permitió objetivar y reconocer sus propios malestares y expectativas. La dirección pudo familiarizarse con algunas de las razones del comportamiento del personal, aspecto muy relevante en vista de que la nueva política implicaba promover su participación en la gestión. Algunas revelaciones importantes fueron la percepción negativa del clima organizacional por parte de los funcionarios, fundamentalmente en lo referente a la comunicación interna, la imagen general de la dirección y el trabajo en equipo, y la falta de una clara visión de la misión institucional. Además, se perfilaron tres grandes desafíos que tenía que enfrentar la dirección para poder continuar con su proyecto: 1) validar la autoridad de las jefaturas y mejorar su capacidad de gestión y comunicación; 2) comprender que parte de la inercia de los funcionarios se debía a la percepción individual de que cada uno estaba haciendo muy buen trabajo, merecía estabilidad laboral y los problemas no eran de su responsabilidad; y 3) verificar que había personal comprometido con el objetivo fundamental de servir al paciente. No obstante, el estudio mostró que el concepto de satisfacer a los usuarios que reinaba en el hospital era principalmente técnico y alejado de su consideración como clientes.

En síntesis, el estudio de clima permitió que la dirección compartiera la percepción de la organización que tenían los funcionarios y se diera cuenta de algunas dimensiones clave que debían enfrentarse para continuar con el proyecto de desarrollo del hospital. También fue relevante que la organización en conjunto, por medio del estudio, participara en la definición de algunos problemas y expresara sus incertidumbres. Aun más importante, se hizo evidente que hacía falta constituir una unidad de recursos humanos que asesorara en estas materias a los jefes correspondientes, lo que implicaba vencer barreras legales y culturales muy arraigadas.

Promoción de una gestión participativa. La difusión de los resultados del estudio de clima generó un período de reuniones y discusiones que duró 2 meses. Los funcionarios expresaron sus ideas sobre cómo imaginaban el hospital "del mañana", cuál debía ser la misión del hospital y cuáles las "reglas del juego" para el cambio. La misión y las reglas se acordaron en una jornada de reflexión de 2 días, "Pensemos juntos el hospital", y se dieron a conocer al país en un acto solemne al que asistieron el ministro de salud y dirigentes de asociaciones del sector de la salud así como representantes de los medios de comunicación. También se dio a conocer el "Acta de acuerdos" emanada de la jornada de reflexión, en la que se declararon la misión del hospital y el nuevo marco de desarrollo institucional.

El Consejo de Desarrollo. Seguidamente se procedió a crear un Consejo de Desarrollo o Directorio del hospital como mecanismo para asegurar una verdadera participación democrática en un clima de confianza, respeto y transparencia, y hacer viables y permanentes los cambios necesarios en el mejoramiento de los servicios. El Consejo cuenta con 12 miembros y es presidido por el director del hospital. Como miembros de la comunidad interna 
participan representantes de los médicos, la Federación Nacional de Trabajadores de la Salud, el Departamento de Enfermería y el grupo de profesionales de colaboración, además de representantes de las jefaturas del área médica, quirúrgica y administrativa. Por la comunidad externa participan representantes de la Universidad de Chile, las organizaciones de recaudación de fondos y las agrupaciones de padres. Este Consejo debe colaborar con la gestión del hospital para asegurar no solo la participación de todos los sectores involucrados en mejorar la atención de salud, sino también la calidad del personal.

Las funciones que se establecieron para el Consejo de Desarrollo fueron 1) definir y evaluar un plan permanente de desarrollo estratégico para el hospital mediante el establecimiento y la evaluación de metas y objetivos; 2) establecer prioridades entre los problemas detectados en la atención de salud de los usuarios; 3) definir pautas para la evaluación cuantitativa (rendimiento) y cualitativa de la gestión hospitalaria en función del buen uso de los recursos; 4) definir e impulsar una política moderna de recursos humanos, a fin de estimular y potenciar las capacidades de los funcionarios; 5) colaborar en el desarrollo de nuevos proyectos y actividades que respondan a las necesidades de los clientes; 6) opinar sobre el fondo y la forma de diversos convenios que se establezcan entre el hospital y terceros (entes públicos, privados sin fines de lucro o comerciales) y velar que cumplan con metas y planes específicos del establecimiento; 7) estimular y canalizar el aporte financiero de instituciones de apoyo, empresas y la comunidad; y también el aporte de ideas e información sobre las necesidades de la propia comunidad a la que sirve el hospital; vigilar el buen nombre e imagen corporativa de la organización y dar cuenta a la comunidad de los resultados de la gestión; 8) promover cambios en la organización dentro de un marco participativo; y 9) velar por que se cumplan la misión institucional y los acuerdos que definen a la organización como un hospital público, preferentemente al servicio de los más pobres. Una vez que se dio este paso hacia el logro de una gestión participativa, el equipo directivo se concentró en formular un plan estratégico con objetivos y metas concretos, para presentarlo al Consejo de Desarrollo.

El plan estratégico. Habiéndose logrado una buena participación democrática en la constitución del Consejo, la generación de una misión institucional y el marco de acuerdos para el cambio, la dirección del hospital trabajó con un equipo compuesto por los subdirectores y otros líderes de la organización para formular un plan estratégico. Teniendo en cuenta tanto las debilidades y deficiencias señala- das en un diagnóstico previo de la situación como las fortalezas de la institución, se propuso un plan que abarca las siguientes ocho áreas prioritarias.

\section{Área de recursos humanos}

Objetivo estratégico: Contar con un sistema de administración de recursos humanos que le permita al hospital disponer oportunamente de personal calificado, motivado y compensado, a fin de lograr las metas de servicio al usuario (véase más adelante).

Metas: Crear una unidad de asesoramiento sobre recursos humanos con componentes de capacitación, psicología, bienestar y personal, que se haga cargo de determinar las políticas de capacitación, selección y reclutamiento, mejora del clima laboral y fomento del bienestar interno.

Ejecutar una política para reconocer moral, social y económicamente a los funcionarios según su desempeño y compromiso con la misión institucional.

Implantar un sistema que permita evaluar objetivamente el desempeño laboral, con referencia a las necesidades de la organización, y que se aplique con uniformidad de criterios.

Hacer un estudio de las cargas de trabajo individuales para conocer las verdaderas necesidades del hospital.

\section{Área de sistemas de información y control de la gestión}

Objetivo estratégico: Contar con un sistema de control de la gestión que asegure el desarrollo de centros de responsabilidad en cada servicio clínico y la evaluación de sus actividades, así como la divulgación apropiada de información sobre los procesos productivos o clínicos.

Metas: Desarrollar centros de responsabilidad en los servicios clínicos, de manera que cada uno de ellos se haga cargo de administrar sus propios recursos.

Tener en funcionamiento un sistema de control de la gestión y de la información.

Iniciar negociaciones o compromisos internos entre la dirección y los centros de responsabilidad sobre el uso de recursos, la producción asistencial y los estándares de calidad.

\section{Área de servicios a usuarios}

Objetivo estratégico: Contar con un sistema integrado y descentralizado de evaluación y mejo- 
ramiento continuo de la calidad de la atención de salud que reciben los clientes del hospital.

Metas: Crear un servicio de atención al cliente, que ofrezca orientación, información y apoyo a los niños y sus familias; que realice encuestas de opinión e incentive y dé respuesta a las opiniones, sugerencias y reclamos de los usuarios del hospital.

Capacitar para reorientar la cultura de la organización hacia un concepto de mejoría continua de la calidad.

Generar y aplicar indicadores de calidad.

\section{Área de la cultura institucional}

Objetivo estratégico: Conseguir que el hospital funcione en todos sus niveles sobre la base de mecanismos de participación estructurados de acuerdo con el trabajo en equipo.

Metas: Mantener funcionando al Consejo de Desarrollo.

Validar un estilo de gestión participatorio y descentralizado a todos los niveles, principalmente en el operativo.

\section{Área de descentralización y autonomía}

Objetivo estratégico: Dentro del marco legal e institucional vigente, concretar un modelo de hospital público, descentralizado y autónomo, mediante un manejo de recursos más descentralizado.

Metas: Lograr que se efectúen los cambios legales y reglamentarios que hagan posible la autonomía de gestión del hospital.

\section{Área de docencia e investigación}

Objetivo estratégico: Establecer un plan que permita incorporar la docencia y la investigación como parte de la gestión general del hospital.

Meta: Llegar a mejorar la integración docenteasistencial.

\section{Área de recursos económicos y manejo financiero}

Objetivo estratégico: Lograr un excedente operacional de $5 \%$ del ejercicio anual por medio de mecanismos adecuados de recuperación de costos, políticas de mercadeo y aprovechamiento máximo de la infraestructura hospitalaria.

Metas: Mejorar la capacidad del hospital para trabajar en competición y complementariedad con el sector privado.
Llevar a cabo estudios de costos y generar políticas de mercadeo.

\section{Área de operaciones}

Objetivo estratégico: Mediante una subdirección operativa, lograr la coordinación entre las áreas médica y de apoyo técnico, básicamente en lo referente a mantenimiento y abastecimiento.

Metas: Profesionalizar el área de abastecimiento, manutención y seguridad en el lugar del trabajo, y asegurar que apoye adecuadamente las actividades del hospital.

Estos ocho objetivos se aprobaron, aun sabiendo que pondrían a prueba la capacidad del hospital y que revelarían todas sus debilidades y factores condicionantes, tanto culturales como de índole jurídica. Añadía otro elemento de tensión el hecho de que el hospital se había constituido en una especie de prueba piloto del cambio en el campo de la salud. Se colocaba así en una especie de vitrina frente a los sectores gremiales y políticos de Chile. Por estas razones se hizo urgente constituir un equipo directivo bien integrado que pudiera liderar con éxito el proceso de cambios y ejecutar el plan estratégico, además de instituir un programa de capacitación intensiva en gran escala como apoyo a la estrategia.

Entre 1995 y 1996 los esfuerzos se centraron principalmente en consolidar cuatro áreas fundamentales: recursos humanos, servicios a usuarios, centros de responsabilidad y nivel de calidad, para lo cual se capacitó prácticamente a todos los funcionarios. Antes de constituir el departamento de recursos humanos, se educó concienzudamente al personal en esa materia, sobre todo para evitar posibles conflictos de poder y falsas expectativas centradas en el bienestar gremial. Asimismo, antes de que se definieran los centros de responsabilidad, se capacitó a los jefes respectivos en aspectos generales de gestión en salud, trabajo en equipo, liderazgo efectivo, mejora continua de la calidad, creación de indicadores y evaluación del desempeño.

\section{Cambios estructurales en cumplimiento del plan estratégico}

En la figura 1 puede apreciarse la estructura directiva del hospital Luis Calvo Mackenna, después de la reestructuración de los servicios clínicos, establecimiento de los centros de responsabilidad y creación del Departamento de enfermería, ubicado bajo la jurisdicción de la subdirección médica. En la práctica, a esta subdirección se le ha hecho funcio- 
FIGURA 1. Estructura directiva del hospital Luis Calvo Mackenna, centro asistencial pediátrico de nivel terciario, Servicio de Salud Metropolitano Oriente, Chile, junio de 1997

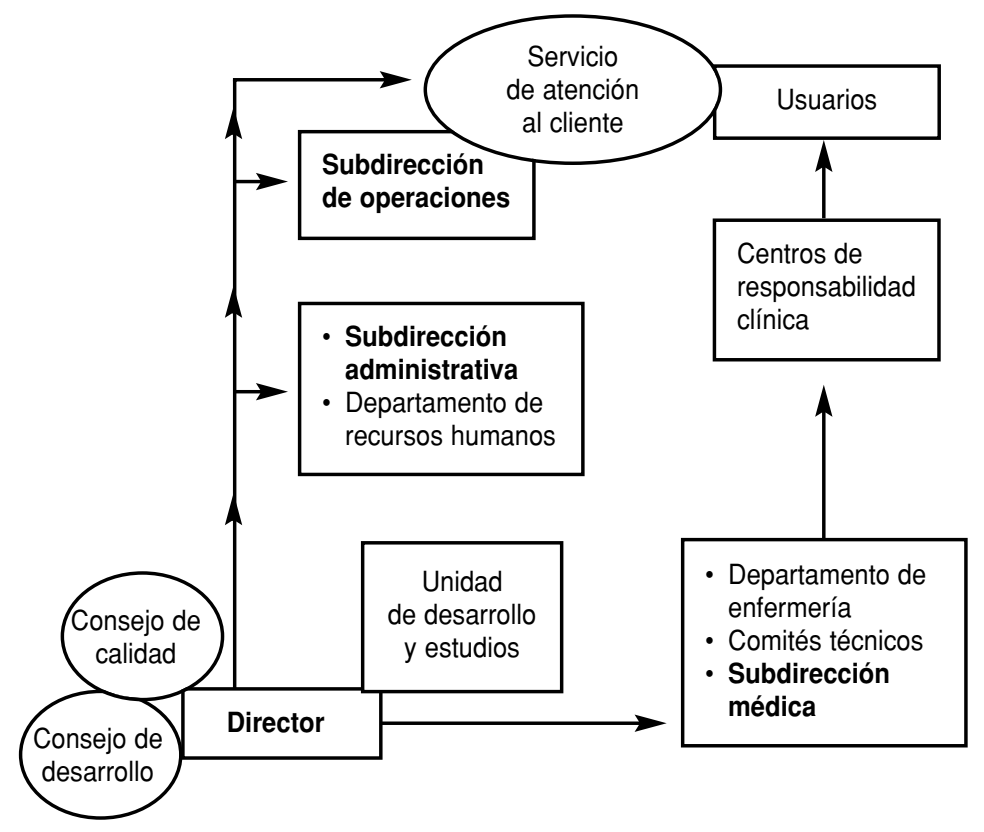

nar más y más como una dirección médica de la cual dependen los centros de responsabilidad y los comités técnicos. Los comités son grupos ampliamente participativos que se ocupan de tomar decisiones sobre nuevas direcciones de desarrollo, inversiones en equipamiento, introducción de tecnología, y otros temas afines. Orgánicamente se relacionan con la subdirección administrativa y su recién creado departamento de recursos humanos, que cuenta con personal profesional experimentado y cuyo objetivo es apoyar una gestión adecuada del recurso humano en cada centro de responsabilidad y en la subdirección de operaciones. Esta nueva subdirección es responsable de toda el área de logística y mantenimiento del hospital. La dirección médica decide los qué y la subdirección decide los cómo.

El director del hospital, que asume progresivamente el papel de gerente de una empresa de servicios, es quien tiene la responsabilidad de mantener y liderar el proceso de cambios en marcha. A su lado tiene dos estructuras de gran importancia: el consejo de desarrollo o directorio del hospital y el consejo de calidad. El primero fija las políticas del hospital a largo plazo; el segundo es un organismo consultivo que asesora y apoya al director en el cumplimiento de políticas, metas y objetivos relacionados con la calidad.

Merecen destacarse dos cambios estructurales cruciales, que contribuyen al cumplimiento del plan estratégico institucional. Uno es el nuevo ser- vicio de atención al cliente, con múltiples funciones decisivas en la reorientación de la organización hacia el cliente. El otro es la creación de la unidad de desarrollo y estudios del hospital. Esta depende del director y está formada por cuatro profesionales y dos técnicos de las áreas de la psicología laboral, apoyo a la gestión, proyectos y planificación, e informática. La unidad tiene la tarea de prestar apoyo interdisciplinario a la ejecución, el monitoreo y la evaluación del plan estratégico.

El equipo directivo definió su propia misión como la de liderar la ejecución del plan estratégico y prestar apoyo a las unidades operativas de manera que estas puedan satisfacer las necesidades de los clientes con una atención de salud eficiente, cálida y de calidad. Se decidió dar prioridad a la atención de los más pobres y de mayor riesgo en un clima de participación y humanidad. El equipo describió su estilo de gestión como participativo, ético, descentralizado, competitivo, multisectorial y comprometido con los principios de calidad y de equidad con eficiencia. Para lograr ese tipo de gestión, declaró el grupo, habrá que conocer las necesidades de los clientes y hacer hincapié en la obtención de resultados; poner énfasis en la capacitación del recurso humano y desarrollar los incentivos y el clima laboral que estimulen su potencialidad; centrarse en las actividades de mayor rentabilidad social; mantener al centro del quehacer el valor y la dignidad del ser humano; usar rectamente los 
recursos que la sociedad ha confiado a la institución; impulsar la creatividad; mantener relaciones con la comunidad, el sector privado y las universidades; y buscar soluciones para los problemas de salud más comunes en el entorno.

En el interior de la organización se han estado llevando a cabo una serie de acciones prioritarias que incluyen estudios a fondo de la estructura organizacional y funciones de cada unidad, comunicación en todos los niveles, equilibrio entre demanda y oferta, manejo de los recursos humanos y otros análisis y diseños de procesos.

\section{CONCLUSIÓN}

En Chile, los hospitales públicos de nivel terciario han tenido una percepción permanente de estar en crisis por su incapacidad de respuesta a una intensa demanda insatisfecha. Esta incapacidad se debe fundamentalmente a falta de recursos pero también a la mala administración de los fondos disponibles, en gran parte motivada por las limitaciones del marco legal y reglamentario vigente, $\mathrm{y}$ al insuficiente hincapié en la atención primaria, mala articulación de la red asistencial y relación poco solidaria entre los subsistemas público y privado.

En junio de 1997, es decir, tres años y medio después del proceso iniciado por el hospital Luis Calvo Mackenna para lograr estabilidad financiera, modernizar su gestión y otros objetivos concretados a mediados de 1996 en su plan estratégico, se evaluaron los cambios efectuados y sus resultados. Sobre esa base se concluyó que ha habido una mejoría significativa en la gestión de los recursos, especialmente de la cama hospitalaria. Ello se ha manifestado en un aumento de la producción medida en egresos hospitalarios y en la capacidad de administrar proyectos específicos. Entre estos figuran el de expansión de la cirugía cardiovascular, disminución de las listas de espera para cirugía y plan especial de invierno para afecciones respiratorias. También se ha consolidado el trabajo participatorio en los diferentes comités descentralizados (farmacia y abastecimiento, por ejemplo) y la gestión se ha hecho mucho más eficiente, pues ha resultado en una mayor disponibilidad de suministros sin aumentar los gastos. Se han determinado las cantidades que se gastan en diferentes servicios y unidades clínicas y la administración de esos recursos se ha descentralizado parcialmente, lo que ha favorecido la consolidación de los centros de responsabilidad previstos en el plan estratégico.

En el hospital se percibe un ambiente de actividad y de liderazgo en un importante proceso de reforma que genera reacciones muy positivas de la comunidad nacional. Aun así, hay ciertos sectores gremiales que ven con temor y desconfianza los aires de cambio. De todos modos, se ha conseguido que la organización avance hacia la transformación prevista e incorpore en su quehacer conceptos como calidad total, contención de costos y trabajo en equipo. Lo más importante es que existe un grupo de personas dedicadas que se han comprometido a luchar por que el hospital Luis Calvo Mackenna se convierta en un hospital público eficiente, con servicios equitativos para todos los sectores de la sociedad chilena y atención especial a los más necesitados.

Manuscrito recibido el 10 de abril de 1996 y aceptado para publicación en versión revisada el 29 de abril de 1996. 
ABSTRACT This article discusses the structural and operational reform process that took place beginning in 1994 in Luis Calvo Mackenna Hospital, a tertiary pediatric care facility run by Oriente Metropolitan Health Center

Structure and human resources in a public hospital in Chile: an experience in conducting changes in Santiago, Chile. The formation of the Chilean health care system is briefly outlined, along with the changes brought about by the military government in 1979 and other transformations introduced by the first democratic government in 1990 and by the second one, in 1994. In spite of Chile's favorable health indicators and of the current government's desire to establish equitable services and to launch a decentralized system, the health care system that was fundamentally modeled in the fifties and sixties has failed to respond successfully to the demographic transition and to the population's new epidemiologic profile. One of the main problems is that services at the secondary and tertiary levels lack what is necessary to respond to the demand. Under a new leadership with an interest in modern administrative methods, clinical services at Luis Calvo Mackenna Hospital were restructured with new departments and units, a study of the organizational climate was carried out, the entire staff was intensively trained so as to generate a spirit of involvement and a new vision of the hospital's mission and teamwork, and a strategic plan was drawn up that is still being implemented. 